\title{
Contenido de fenoles totales y actividad antioxidante del follaje de anís silvestre (Piper auritum Kunth) y su efecto nutracéutico para cerdos en posdestete
}

\section{Total phenolic content and antioxidant activity of wild anise foliage (Piper auritum Kunth) and its nutraceutical effect for pigs in post-weaning}

\author{
Willan Caicedo ${ }^{1,2,3}$, Manuel Pérez ${ }^{1}$, Janeth Sanchez ${ }^{1}$, Alex Flores ${ }^{1,2}$, \\ Elvia Duchitanga ${ }^{1,2}$
}

\section{Resumen}

\begin{abstract}
Se evaluó el contenido de compuestos fenólicos y actividad antioxidante en polvo y extracto cocido del follaje de anís silvestre (Piper auritum Kunth) y su efecto como aditivo fitobiótico para cerdos en posdestete. Se colectaron plantas frescas de anís silvestre y se procesaron en el laboratorio para obtener el polvo y extracto del follaje. Se determinó el contenido de fenoles totales (método Folin-Ciocalteu) y la actividad antioxidante (método ABTS). Se utilizaron 30 lechones castrados Landrace x Duroc de $6 \pm 0.2 \mathrm{~kg}$ distribuidos en tres grupos experimentales: T0 (Dieta basal sin antibiótico promotor de crecimiento) DBSAPC; T1 (DBSAPC + inclusión de $1 \mathrm{~g}$ de polvo/100 g de alimento) y T2 (DBSAPC + inclusión de $10 \mathrm{ml}$ de extracto/100 g de alimento). Se midió el consumo de alimento diario (CAD), ganancia de peso diaria (GPD), conversión alimentaria (CA), peso final (PF) e incidencia de diarreas (ID). El polvo y el extracto presentaron 7.92 $\mathrm{mg}$ ácido gálico/g y $0.75 \mathrm{mg}$ ácido gálico/ml de muestra (fenoles totales) y $0.11 \mathrm{mg}$ TROLOX/g y $0.12 \mathrm{mg}$ TROLOX/ml de muestra (actividad antioxidante), respectivamente. Los cerdos del T2 presentaron mejor CAD, GPD, CA y PF $(\mathrm{p}<0.05)$. Los cerdos de T1 y T2 tuvieron una menor incidencia de ID $(\mathrm{p}<0.05)$. La suplementación dietética con extracto cocido de follaje de anís silvestre en la dieta de cerdos en posdestete mejoró el consumo de alimento, ganancia de peso, conversión alimentaria, peso final y disminuyó la incidencia de diarreas en los animales.
\end{abstract}

Palabras clave: extracto cocido; fitobiótico; lechones; polvo; salud intestinal

\footnotetext{
${ }^{1}$ Departamento de Ciencias de la Tierra, Universidad Estatal Amazónica, Puyo, Pastaza, Ecuador

${ }^{2}$ Granja Agropecuaria Caicedo, Tarqui, Puyo, Pastaza, Ecuador

${ }^{3}$ E-mail: orlando.caicedo@yahoo.es
} 
The content of phenolic compounds and antioxidant activity in powder and cooked extract of wild anise foliage (Piper auritum Kunth) and its effect as a phytobiotic additive for pigs in the post weaning phase were evaluated. Fresh wild anise plants were collected and processed in the laboratory to obtain the powder and foliage extract. The total phenolic content (Folin-Ciocalteu method) and antioxidant activity (ABTS method) were determined. Thirty castrated piglets Landrace $x$ Duroc of $6 \pm 0.2 \mathrm{~kg}$ were distributed in three experimental groups: T0 (Basal diet without antibiotic growth promoter) BDWAGP; T1 (BDWAGP + inclusion of $1 \mathrm{~g}$ of powder/100 $\mathrm{g}$ of feed) and T2 (BDWAGP + inclusion of $10 \mathrm{ml}$ of extract/100 $\mathrm{g}$ of feed). Daily feed consumption (DFC), daily weight gain (DWG), feed conversion (FC), final weight (FW) and incidence of diarrhoea (ID). The powder and extract showed $7.92 \mathrm{mg}$ gallic acid $/ \mathrm{g}$ and $0.75 \mathrm{mg}$ gallic acid $/ \mathrm{ml} \mathrm{sample} \mathrm{(total}$ phenols) and $0.11 \mathrm{mg}$ TROLOX/g and $0.12 \mathrm{mg}$ TROLOX/ml sample (antioxidant activity), respectively. T2 pigs had better DFC, DWG, FC and FW $(\mathrm{p}<0.05)$. Pigs of T1 and T2 had a lower incidence of ID $(\mathrm{p}<0.05)$. Dietary supplementation with cooked extract of wild anise foliage in the diet of pigs during the post weaning phase improved feed consumption, feed conversion, bodyweight gain, final bodyweight and reduced the incidence of diarrhoea.

Key words: cooked extract; phytobiotic; piglets; powder; intestinal health

\section{INTRODUCCIÓN}

El posdestete es una etapa crítica en el desarrollo de los lechones, donde la transición de la dependencia de la leche materna hacia un alimento sólido puede causar disfunciones intestinales e inmunológicas con efectos adversos sobre el crecimiento, la salud e ingesta alimentaria (Reis de Souza et al., 2010).

La porcicultura convencional ecuatoriana es muy demandante en el uso de antibióticos promotores de crecimiento (APC) para prevenir las enfermedades gastrointestinales y mejorar la salud intestinal de los cerdos en posdestete. Sin embargo, la Unión Europea prohibió el uso de antibióticos como promotores de crecimiento en la producción de animales de interés zootécnico desde el año 2003, debido a la presencia de residuos de estos productos en la carne de los animales (CORDIS, 2003). Este entorno dio paso a nuevas investigaciones con alimentos fun- cionales o nutraceúticos, dentro de los que se han incluido los principios bioactivos de plantas conocidos como fitobióticos (Liu et al., 2013). Los fitobióticos, por sus propiedades preventivas y curativas contra enfermedades, tienen un amplio margen de seguridad en su inclusión y no tienen residualidad en el producto final (Mas Toro et al., 2017).

En Ecuador se tiene la planta conocida como anís silvestre (Piper auritum Kunth), que posee un apreciable contenido de metabolitos secundarios con potencial de uso para animales. El follaje de esta planta presenta metabolitos secundarios de naturaleza antioxidante con propiedades antiinflamatorias (Pérez et al., 2012). Estos compuestos polifenólicos tienen funciones trascendentales en la reducción de las especies reactivas del oxígeno (Chahar y Sharma, 2017).

Estudios realizados en conejos con el uso de extracto de anís silvestre en su dieta no encontraron signos de toxicidad o irritabilidad, ni afectaron su peso corporal (López et 
al., 2014). En otros estudios, se han encontrado efectos antibacterianos con el uso de extractos de hojas y raíces de anís silvestre, posiblemente relacionado con la presencia de flavonoides, terpenos y taninos (Reena et al., 2012). Ante estas observaciones, el objetivo de este estudio fue evaluar el contenido de compuestos fenólicos y actividad antioxidante en polvo y extracto cocido del follaje de anís silvestre (Piper auritum Kunth) y su efecto como aditivo fitobiótico para cerdos en la fase de posdestete.

\section{Materiales y Métodos}

\section{Localización}

Las muestras de follaje de anís silvestre (Piper auritum Kunth) se recolectaron en un cultivar de dos años de establecido, en el Rancho Santa Rita, ubicado en la parroquia Tarqui, cantón Pastaza, Ecuador. La zona tiene un clima subtropical húmedo, con precipitaciones anuales entre 4000 y $5000 \mathrm{~mm}$, altitud de $920 \mathrm{msnm}$, con humedad relativa media de $87 \%$ y temperatura mínima y máxima promedio de 20 y $28^{\circ} \mathrm{C}$ (INAMHI, 2014).

El estudio de campo se ejecutó en las instalaciones del programa de porcinos del Centro de Investigación, Posgrado y Conservación Amazónica (CIPCA) de la Universidad Estatal Amazónica (UEA). El CIPCA está ubicado en la Región Amazónica Ecuatoriana (RAE), entre las provincias Pastaza y Napo. En este sector el clima es semicálido o subtropical húmedo, con precipitaciones anuales entre 4000 y $4500 \mathrm{~mm}$, a una altitud de $584 \mathrm{msnm}$, humedad relativa de $87 \%$ y temperatura mínima y máxima promedio de 18 y $36^{\circ} \mathrm{C}$ (Uvidia et al., 2014).

\section{Polvo y Extracto del Follaje de Anís Sil- vestre}

El follaje, una vez recolectado, se trasladó al Laboratorio de Química de la UEA. Las plantas fueron lavadas y puestas a escu- rrir por $1 \mathrm{~h}$. Luego se secaron en estufa Barnstead 3523 por $72 \mathrm{~h}$ a $65^{\circ} \mathrm{C}$. Se molieron en un molino Thomas-Wiley 4 con tamiz de $1 \mathrm{~mm}$, y se colocaron en fundas ziploc estériles por cinco días, hasta su uso. Para la elaboración del extracto cocido, las muestras de follaje se recolectaron diariamente, utilizando $100 \mathrm{~g}$ de follaje fresco/L de agua y llevándolo a ebullición $\left(120^{\circ} \mathrm{C}\right)$. El extracto se dejó enfriar por $1 \mathrm{~h}$ antes de su uso.

\section{Análisis de Laboratorio}

Para determinar el contenido de polifenoles totales se utilizó $40 \mu 1$ de reactivo de FolinCiocalteu (RF-C, Cat. 1090010100) en una dilución 1:1 con agua destilada. La absorbancia se midió a $765 \mathrm{~nm}$ (Stratil et al., 2006).

La capacidad antioxidante del polvo y extracto cocido del anís silvestre se determinó por el método ABTS ácido 2,2 azinobis (3-etilbenzotiazolin-6-sulfónico). La lectura se efectuó a $730 \mathrm{~nm}$ de acuerdo con las sugerencias de Re et al. (1999). Los cálculos se hicieron empleando el modelo matemático de la curva de calibración de TROLOX (patrón de antioxidantes), mediante la fórmula siguiente: $\mathrm{C}=\mathrm{A}-0.7252 /-0.1304$, donde $\mathrm{A}$ es el valor de absorbancia de las muestras y $\mathrm{C}$ es la lectura en el espectrofotómetro.

\section{Animales e Instalaciones}

La investigación se desarrolló según los lineamientos para bienestar animal de la República de Ecuador (AGROCALIDAD, 2017) y el protocolo experimental según las recomendaciones de Sakomura y Rostagno (2007). A partir del destete, 21 días de edad, se hizo la selección y pesaje de los animales. Se utilizaron 30 lechones machos castrados del cruce comercial Largewhite x Duroc, con un peso vivo inicial de $6.0 \pm 0.2 \mathrm{~kg}$. Los lechones se alojaron al azar en corrales individuales de $0.80 \times 1.0 \mathrm{~m}$ durante 14 días. Se trabajó con 10 cerdos por tratamiento y se determinó el consumo de alimento por día (CAD), la ganancia de peso día (GPD), la 
Cuadro 1 Composición de la dieta basal y aporte de nutrientes

\begin{tabular}{lc}
\hline Componentes & Inclusión (\%) \\
\hline Maíz amarillo precocido & 49.984 \\
Leche integral en polvo & 2.000 \\
Harina de soya liofilizada & 18.001 \\
Aceite vegetal & 1.535 \\
Harina de trigo & 10.00 \\
Germen de trigo & 10.00 \\
Carbonato de calcio & 0.401 \\
Fosfato monodicálcico & 2.331 \\
Premezcla Vitamínica Mineral Cerdos ${ }^{1}$ & 0.400 \\
DL-metionina 99\% & 0.283 \\
L-lisina HCL $78 \%$ & 0.663 \\
Cloruro de colina 60\% & 0.200 \\
Antimicótico & 0.050 \\
Cloruro de sodio & 0.500 \\
Almidón & 3.652 \\
Valores calculados ${ }^{2}$ & \\
$\quad$ Energía metabolizable, kcal/kg MS ${ }^{-1}$ & 3350 \\
Proteína cruda, \% & 19.24 \\
Fibra cruda, \% & 2.64 \\
Calcio, \% & 0.70 \\
Fósforo disponible, \% & 0.60 \\
Lisina, \% & 1.53 \\
Metionina + cistina, \% & 0.87 \\
Treonina, \% & 0.75 \\
Triptófano, \% & 0.22 \\
\hline
\end{tabular}

${ }^{1}$ Premezcla suministrada (por kg de dieta seca): Vitaminas; A 1500 UI, D3 300 UI, E 37.5 mg, K 2.5 mg, B1 $1.5 \mathrm{mg}$, B2 $6.25 \mathrm{mg}$, B6 $3 \mathrm{mg}$, B12 $37.5 \mathrm{~g}$, pantotenato de calcio $25 \mathrm{mg}$, ácido fólico $0.5 \mathrm{mg}$, niacina $30 \mathrm{mg}$, biotina $75 \mathrm{~g}$. Minerales: cobalto $0.5 \mathrm{mg}$ (como heptahidrato de sulfato de cobalto), cobre $25 \mathrm{mg}$ (como sulfato de cobre pentahidratado), yodo $1.25 \mathrm{mg}$ (como yodo potásico), hierro $150 \mathrm{mg}$ (como sulfato ferroso), manganeso $100 \mathrm{mg}$ (como óxido de manganeso), selenio $0.5 \mathrm{mg}$ (como selenito de sodio), zinc $0.25 \mathrm{mg}$ (como óxido de zinc)

${ }^{2}$ Calculados según NRC (2012) o determinados

conversión alimentaria (CA), el peso final (PF) y la incidencia de diarreas (ID) (Flores et al., 2015).

\section{Alimentación}

Los tratamientos fueron: T0 (Dieta basal sin antibiótico promotor de crecimiento)-
DBSAPC; T1 (DBSAPC + inclusión de $1 \mathrm{~g}$ de polvo/100 g de alimento) y T2 (DBSAPC + inclusión de $10 \mathrm{ml}$ de extracto/100 g de alimento). La DBSAPC se formuló según los procedimientos de la NRC (2012) (Cuadro 1). El alimento se suministró dos veces al día (08:00 y 15:00), dividido en partes iguales. El agua de bebida estuvo disponible a voluntad. 

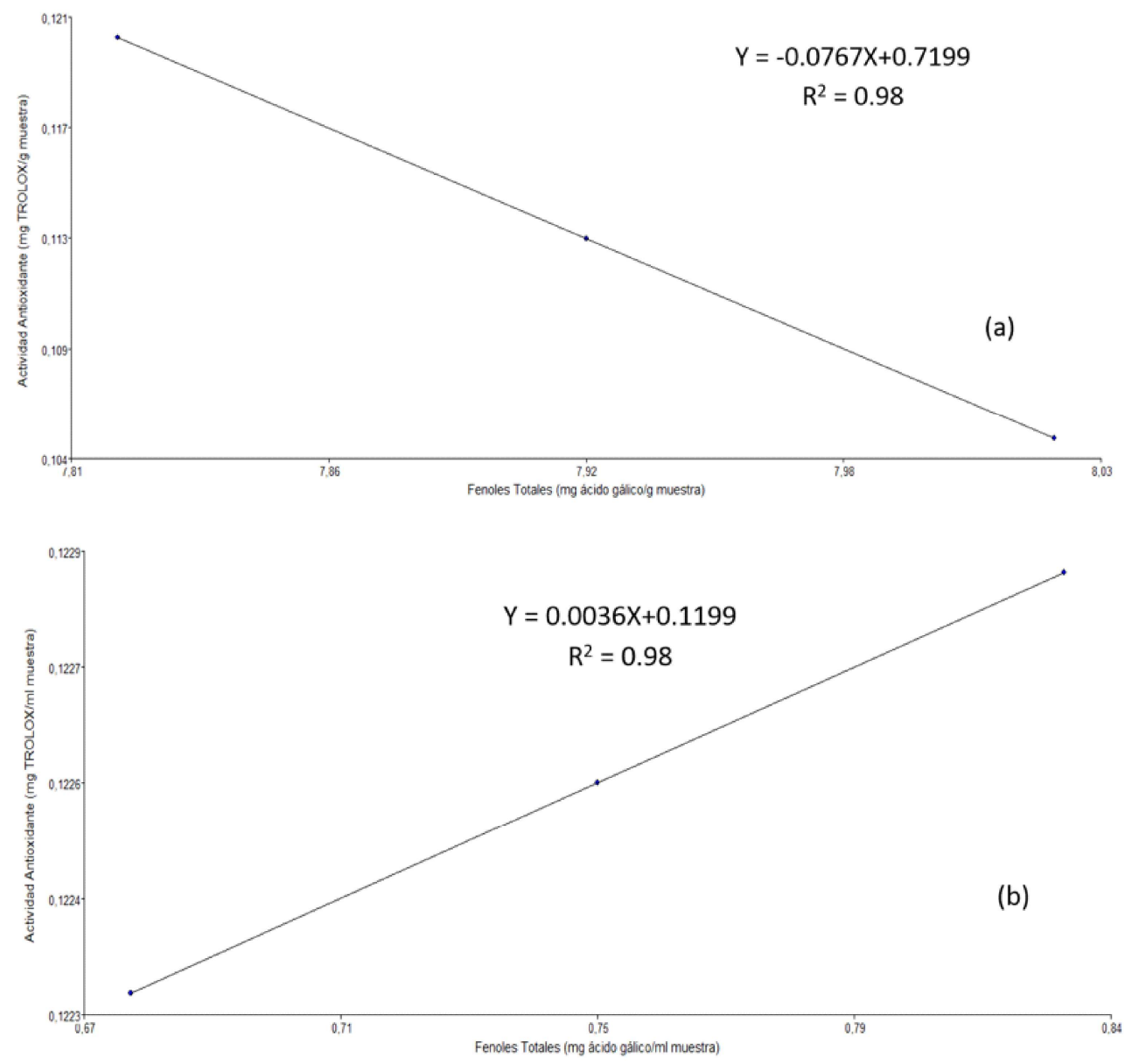

Figura 1. Correlación entre fenoles totales y actividad antioxidante en (a) polvo de follaje y (b) extracto de follaje de anís silvestre (Piper auritum Kunth)

\section{Diseño Experimental}

Para el análisis de fenoles totales y actividad antioxidante se utilizó estadística descriptiva y se determinó la media y desviación estándar de las muestras por triplicado; además, se realizaron pruebas de correlación. Las medias de los indicadores productivos CAD, GPD, CA, PF e ID se contrastaron mediante análisis de varianza y la comparación de medias mediante la prueba de Duncan. Los análisis se hicieron con el programa estadístico Infostat (Di Rienzo et al., 2012).

\section{Resultados}

El polvo de anís silvestre posee $7.92 \pm$ $0.10 \mathrm{mg}$ ácido gálico/g de muestra, $(0.11 \pm-$
$0.01 \mathrm{mg}$ TROLOX/g de muestra) y presentó una correlación negativa $\left(\mathrm{R}^{2}=0.98\right)$ entre fenoles totales y actividad antioxidante (Figura 1a). Por otro lado, el extracto tiene 0.75 $\pm 0.07 \mathrm{mg}$ ácido gálico/ml de muestra), (0.12 $\pm 0.01 \mathrm{mg}$ TROLOX $/ \mathrm{ml}$ de muestra) con una correlación positiva $\left(\mathrm{R}^{2}=0.98\right)$ entre fenoles totales y actividad antioxidante (Figura $1 b$ ).

El tratamiento con extracto de follaje de anís silvestre (T2) presentó el mejor consumo, ganancia de peso, conversión alimentaria, peso final y difirió signifi-cativamente $(\mathrm{p}<0.05)$ de los tratamientos $\mathrm{T} 1$ y T0. La suplementación dietética con los tratamientos $\mathrm{T} 1$ y T2 redujo $(\mathrm{p}<0.05)$ la incidencia de las diarreas en comparación con el tratamiento T0 (Cuadro 2). 
Cuadro 2. Indicadores productivos de cerdos en el posdestete suplementados con polvo y extracto de follaje de anís silvestre (Piper auritum Kunth)

\begin{tabular}{lccccc}
\hline \multirow{2}{*}{ Variables } & \multicolumn{3}{c}{ Tratamientos } & \multirow{2}{*}{ EE } & \multirow{2}{*}{ Valor de $\mathrm{p}$} \\
\cline { 2 - 4 } & $\mathrm{T} 0$ & $\mathrm{~T} 1$ & $\mathrm{~T} 2$ & & \\
\hline Peso inicial, $\mathrm{kg}$ & 6.00 & 6.00 & 6.06 & 0.28 & $\mathrm{p}=0.9888$ \\
Consumo día, kg & $0.28^{\mathrm{c}}$ & $0.31^{\mathrm{b}}$ & $0.34^{\mathrm{a}}$ & 0.03 & $\mathrm{p}<0.0001$ \\
Ganancia de peso diario, $\mathrm{kg}$ & $0.10^{\mathrm{c}}$ & $0.17^{\mathrm{b}}$ & $0.23^{\mathrm{a}}$ & 0.02 & $\mathrm{p}<0.0026$ \\
Conversión alimentaria, $\mathrm{kg} / \mathrm{kg}$ & $3.10^{\mathrm{c}}$ & $1.97^{\mathrm{b}}$ & $1.76^{\mathrm{a}}$ & 0.48 & $\mathrm{p}<0.0024$ \\
Peso final, kg & $7.30^{\mathrm{c}}$ & $8.33^{\mathrm{b}}$ & $8.94^{\mathrm{a}}$ & 0.52 & $\mathrm{p}<0.0127$ \\
Incidencia de diarreas (\%) & $15.00^{\mathrm{b}}$ & $2.23^{\mathrm{a}}$ & $2.15^{\mathrm{a}}$ & 0.67 & $\mathrm{p}<0.0001$ \\
\hline
\end{tabular}

$a, b, c$ Medias con letras distintas en la misma fila son estadísticamente diferentes $(p<0.05)$

T0 (Dieta basal sin antibiótico promotor de crecimiento) DBSAPC; T1 (DBSAPC + inclusión de $1 \mathrm{~g}$ de polvo/100 g de alimento); T2 (DBSAPC + inclusión de $10 \mathrm{ml}$ de extracto/100 g de alimento)

\section{Discusión}

\section{Compuestos Fenólicos y Actividad Antioxidante}

Resultados superiores en cuanto a fenoles totales en follaje seco de anís silvestre fueron reportados por Valdivia et al. (2018) en cultivares producidos en Cuba, posiblemente debido al gran contenido de fenoles ligados a la pared celular que presenta esta especie. Se ha demostrado, asimismo, que la combinación de los solventes etanol-agua $(50: 50 \%)$ y etanol-agua $(70: 30 \%)$ son adecuados para extraer los polifenoles totales del follaje de Piper auritum Kunth $y$ Porophyllum ruderale, seco o fresco (Conde-Hernández y Guerrero-Beltrán, 2014). Por otro lado, se ha reportado que el secado controlado a $65^{\circ} \mathrm{C}$ no causa impacto sobre la reducción del contenido de polifenoles en los vegetales (Cedeño, 2017).

La significativa correlación negativa entre fenoles totales y actividad antioxidante del polvo de anís silvestre pudiera deberse a que no todos los compuestos fenólicos tienen efecto antioxidante (Ramírez et al., 2016). No obstante, los antioxidantes presentes en bajas concentraciones mantienen la capacidad de absorber los radicales de oxígeno (Matthes y Schmitz-Eiberger, 2009; Lutz et al., 2015). Por otro lado, el procesamiento de los vegetales como el escaldado, enlatado, esterilización, congelación y cocción afectan el rendimiento, la composición y la biodisponibilidad de los antioxidantes (Amin et al., 2006; Adebooye y Singh, 2007). Algunos compuestos antioxidantes como el ácido ascórbico, carotenoides y licopenos son muy sensibles al calor y al almacenamiento y se pierden durante el procesamiento (Chipurura

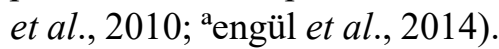

La correlación positiva entre fenoles totales y actividad antioxidante del extracto de anís silvestre pudo deberse a que la cocción controlada impidió la pérdida de metabolitos de importancia como son los flavonoides y algunos taninos (Sharma et al., 2015; Silva et al., 2018). Por otro lado, Laib y Barkat (2018) señalan que las condiciones óptimas de cocción para no afectar el tenor de polifenoles del alimento se establecieron en $92{ }^{\circ} \mathrm{C}$ por 30 minutos. 
Valdivia et al. (2018) evidenciaron que el follaje de Piper auritum Kunth posee buena capacidad antioxidante, mostrando la presencia de terpenos, flavonoides, cumarinas, taninos y glucósidos cardiotónicos, que poseen diversas actividades farmacéuticas. Por otro lado, los aceites esenciales de Piper auritum y P. holtonii han demostrado actividad antifúngica contra Colletotrichum acutatum, C. gloeosporioides y Botryodiplodia theobromae (Pineda et al., 2012). En otro estudio, los extractos etanólicos de hojas y raíces de Piper auritum mostraron efecto antibacteriano contra Staphylococcus aureus ATCC 25923 y Escherichia coli ATCC 25922; así mismo, Valdivia et al. (2018) obtuvieron buenos resultados con los extractos de hojas de Piper auritum Kunth sobre la actividad antibacteriana contra cepas asociadas a mastitis, en tanto que Rojas et al. (2014) encontraron similares resultados contra Pectobacterium carotovorum.

\section{Indicadores Productivos}

Varias plantas y sus extractos han sido utilizadas como aditivos para balanceados por sus propiedades de antioxidantes, efecto antimicrobiano y promotor del crecimiento natural (Hanczakowska et al., 2015). En este sentido, Cheng et al. (2017) utilizaron plantas y sus extractos en las dietas para cerdos, mejorando el rendimiento del crecimiento, la digestibilidad de los nutrientes, la función inmunológica y la calidad de la carne.

El menor consumo de materia seca registrado en T0 y $\mathrm{T} 1$ pudo estar influenciado por la adaptación al cambio de alimento (Reis de Souza et al., 2012) y por el tenor de los taninos presentes en el polvo del follaje de anís silvestre. Los taninos tienen un sabor amargo o astringente que reduce la palatabilidad y, por lo tanto, la ingesta de alimento (Jansman, 1993). Por otro lado, en el proceso de elaboración del extracto se reduce la concentración de taninos (Mamiro et al., 2017), lo cual pudo mitigar su efecto amargo $\mathrm{y}$, consecuentemente, obtenerse una mayor palatabilidad del alimento y un aumento progresivo del consumo de alimento, como fue observado en el grupo de lechones de T2.

La mejor conversión alimentaria se presentó en los lechones del T2, seguido por T1. Lei et al. (2018) reportaron resultados similares al obtener una conversión alimentaria de 1.94. Según Castellanos (2017), las conversiones obtenidas con las dietas de $\mathrm{T} 1$ y $\mathrm{T} 2$ se encuentran dentro de niveles eficientes de conversión alimenticia.

El mejor peso final se obtuvo en forma similar, en los grupos de cerdos de T2 y T1 con relación al grupo control T0. Este resultando es coincidente con el trabajo de Mas Toro et al. (2016) en Cuba empleando dietas compuestas por $1 \%$ de polvo de follaje de guayaba y marañón en cerdos durante el posdestete.

En otro estudio, Zhang et al. (2012) utilizaron un fitobiótico a base de pino coreano (Phytoncide) en cerdos de posdestete y obtuvieron mejoras en la digestibilidad de nutrientes, eficiencia alimentaria, y mayor recuento de lactobacilos a nivel fecal. Estos resultados se deben a que ciertas plantas poseen propiedades antiinflamatorias y astringentes, lo cual resulta efectivo en casos de diarreas o cólicos, además de poseer efectos antibacterianos contra cepas de enterobac-terias manteniendo la salud de la mucosa intestinal de los animales (Martínez et al., 2012).

La mayor incidencia de diarreas en los cerdos del grupo control (T0) puede estar relacionada con la proliferación de E. coli, así como otras bacterias (Lawsonia intracellularis, Clostridium perfringens y Brachyspira pilosicoli), las cuales alcanzan una máxima colonización en el intestino en los cerdos después del destete (Luppi et 
al., 2016; Rhouma et al., 2017). Por otra parte, se pudo evidenciar en T1 y T2 el efecto antidiarreico del polvo y extracto cocido del follaje de anís silvestre; resultado que concuerda con el empleo de plantas medicinales como Anacardium occidentale, Psidium guajava, Morinda citrifolia y M. oleifera en cerdos (Más Toro et al., 2016; ArocheGinarte et al., 2017).

\section{Conclusión}

La suplementación dietética con extracto cocido de follaje de anís silvestre en la dieta de cerdos en posdestete mejoró el consumo de alimento, ganancia de peso, conversión alimentaria y peso final, y disminuyó la incidencia de diarreas.

\section{Agradecimientos}

Se agradece al personal técnico del Rancho Santa Rita y de la Granja Agropecuaria Caicedo, así como del Laboratorio de Química de la Universidad Estatal Amazónica por el apoyo en esta investigación.

\section{Literatura Citada}

1. Adebooye OC, Singh V. 2007. Effect of cooking on the profile of phenolics, tannins, phytate, amino acid, fatty acid and mineral nutrients of whole-grain and decorticated vegetable cowpea (Vigna unguiculatal Walp). J Food Quality 30: 1101-1120. doi: 10.1111/j.17454557.2007.00155.x

2. [AGROCALIDAD] Agencia Ecuatoriana de Aseguramiento de la Calidad del Agro. 2017. Manual de aplicabilidad de buenas prácticas porcícolas. Quito, Ecuador. 127 p. [Internet]. Available in: http:// www.agrocalidad.gob.ec/wp-content/ uploads/pdf/Guia-BPA-publicaciones/ 2017/enero/manual-buenas-practicasporcicolas-24-01-2017.pdf
3. Amin I, Norazaidah Y, Emmy Hainida KI. 2006. Antioxidant activity and phenolic content of raw and blanched Amaranthus species. Food Chem 94: 4752. doi: 10.1016/j.foodchem.2004.10.048

4. Aroche-Ginarte R, Martínez-Aguilar Y, Ayala-González L, RodríguezBertot R, Rodríguez-Fraga Y. 2017. Comportamiento productivo e incidencia de diarrea en cerdos posdestete suplementados con polvo mixto de hojas de plantas con propiedades nutracéuticas. Rev Cien Agric 14: 19-26. doi: 10.19053/ 01228420.v14.n2.2017.7145

5. Castellanos G. 2017. Conversión alimenticia en la granja porcina. [Internet]. Disponible en: http://masporcicultura.com/wp-content/uploads/2017/ nov17/Conversion-alimenticia-cerdosblog.pdf

6. Cedeño S. 2017. Efecto de los diferentes procesos de secado en los compuestos bioactivos de murtilla (Ugni molinae Turcz) y su bioaccesibilidad. Tesis de Maestría. Valdivia, Chile: Univ. Austral de Chile. $40 \mathrm{p}$.

7. Chahar S, Sharma J. 2017. Phytochemical screening, total flavonoid and phenolic content assays and antioxidant activity of Momordica charantia L leaves. Asian J Pharmac Educ Res 6: 60-69.

8. Cheng C, Liu Z, Zhou Y, Wei H, Zhang X, Xia M, Deng Z, Zou Y, Jiang S, Peng J. 2017. Effect of oregano essential oil supplementation to a reduced-protein, amino acid-supplemented diet on meat quality, fatty acid composition, and oxidative stability of Longissimus thoracis muscle in growing-finishing pigs. Meat Sci 133: 103-109. doi: 10.1016/j.meatsci.2017.06.011

9. Chipurura B, Muchuweti M, Manditseraa F. 2010. Effects of thermal treatment on the phenolic content and antioxidant activity of some vegetables. Asian J Clin Nutr 2: 93-100. doi: 10.3923/ ajen.2010.93.100 
10. Conde-Hernández L, GuerreroBeltrán J. 2014. Total phenolics and antioxidant activity of Piper auritum and Porophyllum ruderale. Food Chem 142: 455-460. doi: 10.1016/j.foodchem.2013.07.078

11. [CORDIS] Community Research and Development Information Service. 2003. Un reglamento prohíbe los antibióticos en la alimentación animal como promotores del crecimiento. [Internet]. Disponible en: https:// cordis.europa.eu/article/rcn/20620/es

12. Di Rienzo JA, Casanoves F, Balzarini MG, González, L, Robledo CW. 2012. InfoStat v. 2012. [Internet]. Available in: http://www.infostat.com.ar/

13. Flores L, Elías A, Medina Y, Proaño F, Granizo G, López S, Caicedo W. 2015. Increasing doses of a microbial preparation in the health and productive performance of post-weaning pigs. Cuban J Agric Sci 49: 367-376.

14. Hanczakowska E, Swiatkiewicz M, Grela ER. 2015. Effect of dietary inclusion of a herbal extract mixture and different oils on pig performance and meat quality. Meat Sci 108: 61-66. doi: 10.1016/j.meatsci.2015.05.020

15. [INAMHI] Instituto Nacional de Meteorología e Hidrología. 2014. Anuario Meteorológico. Quito, Ecuador. $28 \mathrm{p}$. [Internet]. Disponible en: http:// www.serviciometeorologico.gob.ec/wpcontent/uploads/anuarios/ meteorologicos/Am\%202011.pdf

16. Jansman A. 1993. Tannins in feedstuffs for simple-stomached animals. Nutr Res Rev 6: 209-236. doi: 10.1079/NRR19930013

17. Laib I, Barkat M. 2018. Optimization of conditions for extraction of polyphenols and the determination of the impact of cooking on total polyphenolic, antioxidant, and anticholinesterase activities of potato. Foods 7: 1-24. doi: 10.3390/foods 7030036

18. Lei X, Yun H, Kim I. 2018. Effects of dietary supplementation of natural and fermented herbs on growth performan- ce, nutrient digestibility, blood parameters, meat quality and fatty acid composition in growing-finishing pigs. It J Anim Sci 17: 984-993. doi: 10.1080/ 1828051X.-2018.1429955

19. Liu Y, Song M, Che TM, Almeida JAS, Lee JJ, Bravo D, Maddox CW, Pettigrew JE. 2013. Dietary plant extracts alleviate diarrhea and alter immune responses of weaned pigs experimentally infected with a pathogenic Escherichia coli. J Anim Sci 91: 5294-5306. doi: 10.2527/ jas.2012-6194

20. López M, García A, Boucourt E, Morejón Z. 2014. Toxicidad aguda tópica e irritabilidad dérmica de la decocción de hojas de Piper auritum Kunth (caisimón de anís). Rev Cubana Plant Med 19: 443-450.

21. Luppi A, Gibellini AM, Gin T, Vangroenweghe F, Vandenbroucke $V$, Bauerfeind R, Bonilauri P, et al. 2016. Prevalence of virulence factors in enterotoxigenic Escherichia coli isolated from pigs with post-weaning diarrhoea in Europe. Porcine Health Manag 2(20). doi: 10.1186/s40813-0160039-9

22. Lutz M, Hernández J, Henríquez C. 2015. Phenolic content and antioxidant capacity in fresh and dry fruits and vegetables grown in Chile. CyTA. J Food 13: 541-547. doi: 10.1080/ 19476337.2015.1012743

23. Mamiro PS, Mwanri AW, Mongi RJ, Chivaghula TJ, Nyagaya $M$, Ntwenya, J. 2017. Effect of cooking on tannin and phytate content in different bean (Phaseolus vulgaris) varieties grown in Tanzania. Afr J Biotechnol 16: 1186-1191. doi: 10.5897/ ajb2016.15657

24. Martínez Y, Soto F, Almeida M, Hermosilla R, Martínez, O. 2012. Metabolitos secundarios y actividad antibacteriana in vitro de extractos de hojas de Anacardium occidentale L. (marañón). Rev Cubana Plant Med 17: 320-329. 
25. Más Toro D, Martínez Y, Rodríguez R, Salazar I, Aroche R, López B, Marcella, D. 2016. Efecto de la suplementación dietética con polvos de hojas de guayaba (Psidium guajava) y marañón (Anacardium occidentale) en el comportamiento productivo y la incidencia de diarrea en cerdos antes y después del destete. Rev Comp Prod Porcina 23: 106-113.

26. Mas Toro D, Aguilar Y, Rodríguez, R, Torres G, Nava O, Olmo C. 2017. Análisis preliminar de los metabolitos secundarios de polvos mixtos de hojas de plantas medicinales. Rev Cubana Plan Med 22: 1-9.

27. Matthes A, Schmitz-Eiberger M. 2009. Polyphenol content and antioxidant capacity of apple fruit: effect of cultivar and storage conditions. J Appl Botany Food Quality 82: 152-157.

28. [NRC] National Research Council. 2012. Nutrient requirements of swine. $11^{\text {th }}$ ed. Washington DC, USA: National Academies Press. 400 p.

29. Pérez RM, Flores LB, Neira AM. 2012. Evaluation of the antioxidant and anti-glication effects of the hexane extract from Piper auritum leaves in vitro and beneficial activity on oxidative stress and advanced glycation endproduct-mediated renal injury in streptozotocin-treated diabetic rats. Molecules 17: 11897-11919. doi: 10.3390/ molecules 171011897

30. Pineda R, Vizcaíno S, García CM, Gil JH, Durango DL. 2012. Chemical composition and antifungal activity of Piper auritum Kunth and Piper holtonii C. DC. against phytopathogenic fungi. Chilean J Agric Res 72: 507-515. doi: 10.4067/S0718-58392012000400008

31. Ramírez M, Alvarado M, Rodríguez JG 2016. Correlación de polifenoles totales, actividad antioxidante y potencial reductor de plantas nativas del semidesierto de Coahuila. Inv Desarrollo Cienc Tecnol Alimentos 1: 151-156.
32. Re R, Pellegrini N, Proteggente A, Pannala A, Yang $M$, Rice-Evans $C$. 1999. Antioxidant activity applying an improved ABTS radical cation decolorization assay. Free Radic Biol Med 26: 1231-1237. doi: 10.1016/s08915849(98)00315-3

33. Reena J, Nidhi K, Vijay K, Amit KS, Swati G, Shrotri CK. 2012. In vitro antibacterial potential of different extracts of Tagetes erecta and Tagetes patula. J Natural Sci Res 2: 84-90.

34. Reis de Souza T, Mariscal G, Escobar K. 2010. Algunos factores fisiológicos y nutricionales que afectan la incidencia de diarreas post destete en lechones. Vet México 41: 275-288.

35. Reis de Souza T, Mariscal G, Escobar K, Aguilera A, Magné A. 2012. Cambios nutrimentales en el lechón y desarrollo morfofisiológico de su aparato digestivo. Vet México 43: 155-173.

36. Rhouma M, Fairbrother JM, Beaudry F, Letellier A. 2017. Post weaning diarrhea in pigs: risk factors and noncolistin-based control strategies. Acta Vet Scand 59: 1-19.

37. Rojas MM, Corzo M, Sánchez Y, Brito D, Montes de Oca II, Martínez Y, Pino O. 2014. Actividad antibacteriana de aceites esenciales sobre Pectobacterium carotovorum subsp. Carotovorum. Rev Protección Veg 29: 197-203.

38. Sakomura N, Rostagno, H. 2007. Métodos de pesquisa em nutrição de monogástricos. Jaboticabal, Brasil: FUNEP. 283 p.

39. "engül M, Yildiz H, Kavaz A. 2014. The effect of cooking on total polyphenolic content and antioxidant activity of selected vegetables. Int J Food Properties 17: 481-490. doi: 10.1080/ 10942912.2011.619292

40. Sharma K, Ko EY, Assefa AD, Ha S, Nile SH, Lee ET, Park SW. 2015. Temperature-dependent studies on the total phenolics, flavonoids, antioxidant activities, and sugar content in six onion varieties. J Food Drug Analysis 23: 243252. doi: $10.1016 /$ j.jfda.2014.10.005 
41. Silva MO, Brigide P, De Toledo NMV, Canniatti-Brazaca S. 2018. Phenolic compounds and antioxidant activity of two bean cultivars (Phaseolus vulgaris L.) submitted to cooking. Braz J Food Technol 21: 1-8. doi: 10.1590/19816723.7216

42. Stratil P, Klejdus B, Kubán V. 2006. Determination of total content of phenolic compounds and their antioxidant activity in vegetables - evaluation of spectrophotometric methods. J Agric Food Chem 54: 607-616. doi: 10.1021/jf052334j

43. Uvidia H, Buestán D, Leonard I, Benítez D. 2014. La distancia de siembra y el número de estacas en el esta- blecimiento del Pennisetum purpureum. REDVET 15(7). [Internet]. Disponible en: https://www.redalyc.org/pdf/636/ 63632383004.pdf

44. Valdivia A, Rubio, Y, Camacho C, Brea O, Matos M, Sosa M, Perez Y. 2018. Propiedades fitoquímicas y antibacterianas de Piper auritum Kunth. Avances Inv Agropec 22: 77-89.

45. Zhang S, Jung JH, Kim HS, Kim BY, Kim IH. 2012. Influences of phytoncide supplementation on growth performance, nutrient digestibility, blood profiles, diarrhea scores and fecal microflora shedding in weaning pigs. AsianAustralas J Anim Sci 25: 1309-1315. doi: 10.5713/ajas.2012.12170 